\title{
Legal Accelerators and Brakes for Deployment of Automated Vehicles
}

\author{
Karlyn D. Stanley, Ellen Partridge and Frank Douma
}

\begin{abstract}
This chapter will review three questions that prompted significant discussion at the 2014 Symposium on Vehicle Automation sponsored by the Transportation Research Board (TRB) and the Association for Unmanned Vehicle Systems International (AUVSI) as potential accelerators or brakes for deployment of automated vehicles: (1) Where are uniform laws needed? (2) What deployment will come first and will it be evolutionary or revolutionary? (3) How should tests be devised for ratings or certification? Participants in the "Legal Accelerators and Brakes" session noted that the legal environment does not appear to be the obstacle, or "brake" to autonomous vehicle deployment that many fear it will be. Greater uniformity in operational laws, such as tailgating and distracted driving, as well as in safety testing standards, could potentially accelerate deployment. Participants in the session concluded that key privacy and security questions will be informed by legal developments that are not unique to driving.
\end{abstract}

Keywords Legal framework - Automated driving • Evolution vs. Revolution

Disclaimer The views, opinions and statements contained in this article are solely those of the author and do not represent the official policy or position of the Department of Transportation or of the Office of the Assistant Secretary for Research and Technology.

\footnotetext{
K.D. Stanley $(\bowtie)$

RAND Corporation, 1200 South Hayes Street, Arlington, VA 22202-5050, USA

e-mail: kstanley@ rand.org

E. Partridge

U.S. Department of Transportation, 1200 New Jersey Avenue Southeast, Washington

DC 20590, USA

e-mail: ellen.partridge@dot.gov

F. Douma

University of Minnesota, Minneapolis, MN 55455, USA

e-mail: douma002@umn.edu

G. Meyer and S. Beiker (eds.), Road Vehicle Automation 2,

Lecture Notes in Mobility, DOI 10.1007/978-3-319-19078-5_9
} 


\section{Introduction}

Recent developments in automated vehicle technologies, along with the publicity they have generated, have created considerable excitement about the opportunities for increased safety, capacity, mobility and access. These advances have also generated questions about the legality of operating these "self-driving" vehicles on public roadways, which has led to a number of states and Washington, D.C. passing specific legislation authorizing operation of these vehicles within their jurisdiction. Lead among these, California is in the final stages of promulgating rules to implement the law passed by its legislature in 2013. In the meantime, the National Highway Traffic Safety Administration (NHTSA) has issued a policy statement regarding its potential role in regulating the safety aspects of these technologies [1].

This activity has not gone unnoticed in the legal community. In particular, these activities were in the forefront of those participating in the "Legal Accelerators and Brakes" session at the 2014 Symposium on Vehicle Automation sponsored by the Transportation Research Board (TRB) and the Association for Unmanned vehicle Systems International (AUVSI) in San Francisco. This group was charged with focusing on the following core issues:

- The mode most likely to lead deployment of these technologies in the current regulatory environment;

- The role "infotainment" applications might play in driving or delaying deployment;

- Whether uniform laws would help or hinder deployment;

- What opportunities and threats exist from the increasing amounts of data generated and used by these technologies; and

- How liability questions might be handled.

This chapter will review three questions that prompted significant discussion as potential accelerators or brakes: (1) Where are uniform laws needed? (2) What deployment will come first and will it be evolutionary or revolutionary? (3) How to devise tests for ratings or certification?

\section{Where Are Uniform Laws Needed?}

The item that emerged as a key "Accelerator" was the role uniform laws could play. After several presentations and group discussions, the participants concluded that the current processes of different states and agencies developing rules independent of each other was not an ideal setting for bringing these technologies to market, and realizing their benefits, as quickly as possible. However, the group was also cognizant of the risks inherent in regulations being issued too soon, i.e., before the technologies have been fully tested, modified, and matured. Consequently, the recommendation was that we should consider where we need uniformity to ensure 
smooth operations and deployment across state lines and across manufacturers of autonomous vehicles.

Providing a uniform regulation of interstate commerce has been a key clause in introducing federal regulations [2], and in promoting development of the U.S. economy. Further, the legal literature has plenty of examples of attorneys and others seeking to reconcile different regulatory approaches to various issues, ${ }^{1}$ and at least one commentator has already called for increased activity and deference to NHTSA in developing safety standards for these technologies [3]. As is often the case in dealing with advanced technologies, however, providing a clean and acceptable set of regulations is more easily said than done, as these technologies are difficult to define and do not fit neatly into the jurisdiction of only one or two existing regulatory agencies.

However, it is worth first asking whether complete uniformity is desirable, let alone possible. This section will briefly discuss the wide scope of areas self-driving vehicles may affect, and then comment on the discussions about liability, security and privacy.

\subsection{Areas for Potential Uniformity}

Self-driving vehicles raise a number of legal issues. Privacy, security and liability have figured most prominently. In particular, the role of law and policy in addressing the transition from human control in NHTSA's levels 2 and 3 to completely self-driving vehicles in level 4 is unclear. There is widespread agreement that ensuring consistent communication standards between vehicles and other parts of the infrastructure is essential. Legal regimes that are typically the province of state and local regulations, such as driver licensing and laws on operational requirements - e.g., tailgating and distracted driving — will need further examination to see where and how uniformity may be needed.

Participants in the conference session identified several areas where laws need to be brought into uniformity to allow large-scale adoption of autonomous vehicles. For example, although the "platooning" of autonomous vehicles in commercial trucking fleets was discussed as being one of the first large-scale areas of potential deployment, participants in the session identified the need for uniform laws concerning minimum vehicle distance following requirements. Currently, "platooning" could violate "tailgating" laws in some U.S. states, as well as in Europe. In addition, new uniform laws or regulations concerning certification of commercial vehicles that are properly equipped to participate in "platooning," as well as uniform enforcement measures to prevent "free riders" from joining a commercial vehicle platoon may be needed.

\footnotetext{
${ }^{1}$ The work of the National Conference of Commissioners on Uniform State Laws is one of the most prominent efforts in this area.
} 
In the areas of liability, security and privacy, legal developments outside the realm of autonomous vehicles will lead developments in those arenas. Because those developments will inform law relating to autonomous vehicles, progress in each of these areas makes it more likely that lack of uniformity will not impede deployment of these technologies.

\subsubsection{Liability}

One significant question that consistently arises in the discussion of self-driving vehicles is that of liability when something inevitably goes wrong. Given the increased activities conducted by the vehicle, it would appear that personal liability is being replaced by products liability. While this may be the case to some extent, some crashes will still involve human decisions and actions, and some damage will be inflicted upon vehicles by something besides another vehicle (such as a falling tree or hailstone), which indicates that some level of personal insurance coverage, if not explicitly liability coverage, will be necessary long into the future. Regardless, current law provides tests and processes for resolving these issues, and has done so for years without complete uniformity. ${ }^{2}$ In addition, insurance markets have developed to provide products for technology risks. Consequently, there is currently little need for new law, or resolving differences in existing law, although the situation could change if evidence develops that liability concerns are significantly dampening the introduction of life-saving technologies.

\subsubsection{Security}

A second major question is the use and protection of the data created. Data breaches that leak a driver's travel history into unauthorized hands could create significant skepticism regarding whether the safety and other benefits outweigh the risks to preserving an individual's privacy. This could lead to significant delay in, if not detrimental alteration of, how these technologies are deployed. However, the question of protecting data is not unique to self-driving vehicles, and measures to protect knowledge about a person's personal whereabouts are already under consideration in several states and countries. ${ }^{3}$ As these efforts mature, they will provide

\footnotetext{
${ }^{2}$ For example, a products liability defense attorney at the TRB/AUVSI meeting pointed out that two different tests (risk-benefit and consumer expectation) co-exist in the United States without creating significant legal confusion.

${ }^{3}$ For example, California's Senate Bill No. 1298, which would add language to the California Vehicle Code, states: ' $(\mathrm{G})$ The autonomous vehicle has a separate mechanism, in addition to, and separate from, any other mechanism required by law, to capture and store the autonomous technology sensord ata for at least 30 seconds before a collision occurs between the autonomous vehicle and another vehicle, object, or natural person while the vehicle is operating in autonomous mode.' http://www.leginfo.ca.gov/pub/11-12/bill/sen/sb_1251-1300/sb_1298_bill_20120925_chaptered.pdf.
} 
a significant, and useful, roadmap to deployment, without the need for specific common legislation across the 50 states.

Security against malicious hacking that might intentionally cause accidents is another facet of the security concern. It, too, will be informed by legal and technological developments in other arenas, such as banking and finance, where countermeasures to hacking lead the way.

\subsubsection{Privacy}

Related to the security question, is that of privacy, i.e., who can see my data, what can they do with it, and how can I protect against someone else accessing my data in the first place? Data of particular interest for self-driving vehicles relates to an individual's current location and history of movement. Misuse of data could destroy consumer confidence in the technology.

In 1983, the Supreme Court held that a person does not have an expectation of privacy in his or her movement on the public thoroughfare. Since then, however, the amount of data that is created and may be available, has increased dramatically, creating many more opportunities for data to be shared and used far beyond the purpose of helping "drive" the vehicle, and it is not clear that the wide variety of state level protections provide any direction or clarity for developers of autonomous vehicles. Fortunately, the Supreme Court does seem to indicate an openness to restricting who can access this information. In U.S. v. Jones [5], a unanimous Court held that physical placement of a tracking device on a vehicle required a search warrant, and concurring opinions noted that tracking the vehicle's movements over 28 days may have also been unreasonable. The Court extended this protection to cell phone data as well in another unanimous decision [6]. Consequently, while control over personal data will likely remain a key obstacle, these cases appear to be setting some useful direction.

\section{What Deployment Will Come First? Will It Be Evolutionary or Revolutionary?}

Participants in the "Accelerators and Brakes" session at the TRB/AUVSI conference concluded that initial deployment of fully autonomous vehicles was most likely to occur on closed campuses where vehicles would be traveling at slow

(Footnote 3 continued)

See also Alliance of Automobile Manufacturers (“Auto Alliance”) Privacy Principlesf or Vehicle Technologies and Services,http://www.autoalliance.org/index.cfm?objectid=865F3AC0-68FD11E4-866D000C296BA163 last accessed 29 May 2015. 
speeds. This conclusion was in part because uniform regulations about the use of autonomous vehicles on public highways would not have to be in place.

The scenario for deployment of autonomous passenger vehicles on shared roadways was quite different. The adoption of autonomous passenger vehicles was described as being more evolutionary than for commercial vehicles. "Infotainment," the blend of information provided by telematics and in-vehicle entertainment systems, was identified as being a key "accelerator" of autonomous passenger vehicles. Obviously, one of the great attractions of autonomous vehicles for consumers will be the opportunity to do something other than drive the vehicle, whether that means text messaging, watching a video, or talking on the phone. However, some infotainment systems may violate the "distracted driving" laws of many U.S. states. ${ }^{4}$ To accelerate the deployment of autonomous passenger vehicles, state distracted driving laws need to be reviewed and brought into uniformity that appropriately permits telematics and infotainment systems. In Europe as well as the United States, both "platooning" and "infotainment" may require changes to existing laws and greater uniformity of laws.

"Platooning" refers to an arrangement of vehicles on the roadway where one lead vehicle with a driver is followed very closely by a fleet of autonomously-driven vehicles. The autonomous vehicles are "following the leader" and gain significant benefits from this arrangement, including fuel savings (5\% for the lead vehicle and $10 \%$ for following vehicles, according to fleet management experts at the conference). Platooning may also impact two other key areas of the financial model for commercial trucking fleets: the cost of tires and driver fatigue. Platooning can reduce driver fatigue in all of the participating vehicles except the lead vehicle and a platoon's consistent road speed can reduce tire wear. Driver shortage is also an issue for commercial fleet management. Platooning allows lower cost, less experienced drivers to follow the lead vehicle, producing an estimated savings of $8-10 \%$, according to experts who participated in the break-out session. Fleet management experts who participated in the "Accelerators and Brakes" session suggested that these financial numbers, which quantify the benefits of automation, would drive the adoption of autonomous vehicles by commercial trucking fleets.

Fleet management experts described different "tailgating" laws in the U.S. and Europe as constituting a potential "brake" on the use of platooning by commercial trucking fleets. For example, California requires a minimum following distance that is "reasonable and prudent" given speed and road conditions, which is usually interpreted as a following distance of $3 \mathrm{~s}$ behind another vehicle. A 3-s following distance would not work for an autonomous vehicle in a platoon, where the dynamic of closely following a lead vehicle produces fuel efficiencies. Experts at the break-out session estimated the following distance for platooning vehicles as 4

\footnotetext{
${ }^{4}$ Some of these activities may be illegal in the context of individual state "distracted driving" laws. Currently, 44 states, D.C., Puerto Rico, Guam, and the U.S. Virgin Islands ban text messaging for all drivers. Fourteen states, D.C., Puerto Rico, Guam, and the U.S. Virgin Islands prohibit all drivers from using handheld cell phones while driving. www.distraction.gov (last accessed $11 / 25 / 14)$.
} 
$16 \mathrm{~m}$. Since commercial trucking fleets often travel between U.S. states and between European countries, in order for commercial trucking fleets to deploy platooning effectively, these laws may need to be modified to permit platooning. Moreover, with uniform operating requirements, commercial trucking fleets can adopt standard procedures for platooning that will improve safety and assure the financial benefits of platooning.

Fleet management experts explained that the challenge will be to prevent commercial vehicles that are not properly equipped with platooning technology from joining roadway platoons. There may be a need to certify that commercial vehicles meet certain technology and performance standards for platooning, as well as a method to determine that vehicles that wish to join a platoon are certified. In addition, fleet managers explained, there will need to be a way to prevent "free riders" from joining a commercial trucking platoon. These operational requirements may lead to a need for uniformity across all 50 states and in other countries where the vehicles share a common market.

It is more difficult to quantify the return on investment for autonomous vehicles in the passenger fleet, and that may make deployment more incremental. Adaptive cruise control, parking assistance, and forward crash avoidance may become common in passenger vehicles long before the adoption of fully autonomous passenger vehicles takes place on a large scale. However, in the future, autonomous passenger vehicles may provide benefits such as greater safety, fuel savings, reduced highway congestion, and reduced carbon emissions [7].

\section{How to Devise Tests for Ratings or Certification? How Can Performance Measures Be Set to Have a Safety Baseline and Still Encourage Innovation?}

The traditional federal role in transportation safety has been to regulate the vehicle while the state and local role has been to regulate the driver. Automated vehicles introduce a number of complexities into that system. For example, these technologies bring the vehicle much closer to being the actual driver, and the vehicle's systems themselves are complex. The vehicle responds to a host of variables as it senses conditions (with multiple sensors and visual interpretation software), makes decisions (comparing the information from the various sensors as it is programmed to do), and takes action. It may even have algorithms that learn and adapt to changing conditions. It may be programmed to weight variables in making value decisions.

The interaction between the driver and a vehicle with new technologies and capabilities may mean that driver licensing has to be more detailed about the knowledge required to operate a particular vehicle. Rather than one all-purpose driver's license, multiple tests may be required to assure a driver's basic familiarity with electronic assist features as well as required interactions between drivers and vehicles. 
For the vehicle itself, safety will be paramount to consumer acceptance. Safety assurance can come from tests, certifications, and/or regulations. These are in addition to the developmental testing that a manufacturer would undertake. As noted above, a few states are in the preliminary stages of regulation and the federal government (NHTSA) has set forth a policy document, but has not begun formal rulemaking. Too early regulation could discourage innovation with insurmountable constraints or put in place requirements for sub-optimal technology.

\subsection{Why Have Vehicle Tests?}

Tests are not the same as regulations. Meeting testing requirements is one element in assessing performance and defending against potential liability. There may be a technological benefit from a more uniform fleet, with clearer expectations for manufacturers and suppliers, and safer vehicles with consumers knowing what to expect. Other parts of the overall transportation system - traffic lights, highway line markings, maps and digital infrastructure - may function better with a more uniform vehicle that other products are designed to complement.

From a manufacturer perspective, demonstrating compliance with testing requirements - voluntary or mandatory - is evidence that influences whether it is found to have exercised the proper standard of care if a crash occurs and a lawsuit follows. A manufacturer's own internal testing regime, with quality assurance inspections and gathering and preserving data, is critical for defending the design decisions that were made.

\subsection{How Have Tests Traditionally Been Done?}

Vehicle safety tests have been designed to be objective performance tests. Conditions for the test are specified in great detail and the vehicle response is measured and compared to performance standards. An example is NHTSA's frontal crash test, often featured on the nightly news as new vehicles are tested. Two crash test dummies are placed in the front seats and belted in. The vehicle is then crashed into a fixed barrier at 35 miles an hour and the damage to the crash dummies is measured. Instruments measure the force of impact to each dummy's head, neck, chest, pelvis, legs and feet. The vehicle is then given from one to five stars to provide consumers with information about crash protection beyond what is required by federal law. Every detail of the dummy and the test is prescribed, down to the dummy's shoe size. 


\subsection{What Are the Potential Lessons from Traditional Tests?}

Automated vehicle technology changes how we think about safety and what safety measures need to be adopted. It moves the standard from crash worthiness to crash avoidance. Past lessons and concepts are still useful as a starting point, but there are hurdles to overcome in devising appropriate tests. New factors include:

- Potentially infinite variables. The car's sensors are faced with distinguishing whether an object in the road is a ball, a dog, or a small child. Its decision systems may note that if it is a ball, then a child may follow.

- Increased role of software. The software is complex and if something goes wrong, it is not always easy to determine what went wrong.

Tests have dealt with similar issues before. Using the crash testing example, there can be multiple variables. Crash testing can occur in various configurations of number of vehicles, speed, direction, and angle of the vehicles. The set of tests is designed to encapsulate performance and come to conclusions about vehicle performance safety in ways that happen in real world.

For a software example, electronic stability control testing tests the software's ability to do what it is intended to do. Following the method of crash testing, the vehicle is exposed to conditions where software needs to intervene or it will fail the test. This tests the ability of the software to sense, think, process, and effectuate a decision. While there may be more variables in crash avoidance testing than in crashworthiness testing, the basic method is to set performance criteria, choose variables, and evaluate software intervention and decision.

The issues and interactions are more complex, but past tools, methods, and concepts that have been developed and researched provide a platform to develop tests to evaluate safety performance for new technologies. Public acceptance of automated vehicles may be enhanced as tests are developed.

\subsection{What Are the Drawbacks to Tests?}

Tests encourage uniformity. Liability may be less likely to be found with uniformity, but is it good to have a homogeneous fleet in a climate of innovation? Timing is key. As with regulations, premature public test protocols may crowd out innovation.

While cars are improved based on the tests, some part of the improvement may come from "teaching to the test." When a new test is introduced, in many cases the ratings quickly move from "mostly poor to nearly $100 \%$ good." A speaker from a firm that develops and conducts vehicle tests described a new test introduced in 2012 that initially show ratings that distinguished among vehicles; later, it likely will not. Manufacturers tune the system to perform better, with respect to a particular test. 
Tests have been developed for autonomous braking systems, with the expected result that manufacturers will do better now that they have a target to shoot toward. One test showed dramatically different test results in a three-month period from April to July 2014.

The question was also raised of how do we know that the test is testing the right thing? Tests might mislead the consumer. In some instances, designing to a test may not encourage safety. Oversensitive braking might pass a test for speed of braking with flying colors, but may not be best for safety. This could occur if vehicles behind it do not have time to stop without colliding.

Tests for software will have to devise a number of scenarios and assess acceptable responses. Testing software may be more like grading an essay than a multiple-choice test, and in a language that is not your native tongue. The difficulty of the task does not make it an impossible challenge. Rather it drives home the fact that great care needs to be given to when rating tests should be introduced, what functionalities should be tested, and how.

\section{Conclusion}

This chapter examined potential accelerators or brakes for the deployment of autonomous vehicles, including: whether uniform laws are needed; which mode of transportation will deploy autonomous vehicles first, including whether the deployment will be evolutionary or revolutionary; and how tests may be devised for ratings or certifications of autonomous vehicles.

Generally, the participants in the break-out session concurred that it was important to consider where uniformity was needed to ensure smooth operations and deployment across state lines and across manufacturers of autonomous vehicles. Privacy, security, and liability figured prominently in the discussions. In terms of liability, current law provides tests and processes for resolving liability issues without complete uniformity. Consequently, although liability is a key issue, there appears to be little immediate need for new laws, or resolving differences in existing laws. Participants in the break-out session noted that the issue of protecting personally identifiable information is not unique to autonomous vehicles, and measures to protect information about a person's location, for example, are already under consideration in several states and countries. Therefore, at the current time, specific common legislation in all 50 states about autonomous vehicle data is not required for deployment. Consumer anxiety over control of personal automotive data is likely to be a key issue for deployment of autonomous vehicles, but attempts to develop uniformity of state privacy laws could have consequences far beyond self-driving vehicles.

Participants and transportation experts in the break-out session identified several areas where uniformity of laws would be useful for autonomous vehicle deployment. In Europe as well as the United States, both platooning of autonomous vehicles and infotainment may require changes to existing operational laws and 
greater uniformity of laws. Fleet management experts described different "tailgating" laws in the United States and Europe as constituting a potential "brake" on the use of platooning by commercial trucking fleets. Similarly, although infotainment may accelerate the deployment of autonomous vehicles, greater uniformity of state "distracted driving" laws may be necessary to achieve this result. There may also be a need for uniformity of certification standards for platooning vehicles across all 50 states and in Europe.

Participants in the break-out session concluded that initial deployment of fully autonomous vehicles was most likely to occur on closed campuses where vehicles would be traveling at slow speeds, in part because uniform laws about the use of autonomous vehicles on public highways would not have to be in place. Fleet management experts suggested that financial data that quantified the benefits of automation would drive the adoption of autonomous vehicles by commercial trucking fleets. Platooning of commercial vehicles was identified as one of the first large-scale areas of potential deployment. The scenario for deployment of autonomous passenger vehicles was described as much more evolutionary than for commercial vehicles.

Autonomous vehicles introduce new complexities into the current framework of state and federal vehicle and driver regulation, testing, and certification. For example, autonomous vehicles will have new, more complicated technology. Rather than one all-purpose driver's license, multiple tests may be required to assure a driver's basic familiarity with electronic assist features, as well as required interactions between drivers and vehicles. Participants in the break-out session concurred that autonomous vehicle safety will be paramount to consumer acceptance. Safety assurance can be derived from tests, certifications, and regulations. In a discussion of new factors that may need to be included in testing, two issues were highlighted: first, the potentially infinite testing variables that could arise from a vehicle's sensors, and second, the increased role and complexity of on-board vehicle software. A transportation expert in the break-out session noted that although the testing issues for autonomous vehicles may be more complex, past tools, methods, and concepts provide a platform to develop tests to evaluate the safety performance of new technologies. To encourage innovation, critical aspects of any rating or certification requirements for autonomous vehicles will be when tests should be introduced, what functionalities should be tested, and how they should be tested.

Regulators and legislators continue to try to anticipate problems that may occur with automation and to assess whether the benefits of automation are so significant as to compel actions to accelerate its adoption. Overall, the legal environment does not appear to be the obstacle, or "brake" to autonomous vehicle deployment that many fear it will be. Greater uniformity in operational laws, such as tailgating and distracted driving, as well as in safety testing standards, could potentially accelerate deployment. Key privacy and security questions will be informed by developments in the law that are not unique to driving. 


\section{References}

1. National Highway Traffic Safety Administration. Preliminary statement of policy concerning automated vehicles. http://www.nhtsa.gov/staticfiles/rulemaking/pdf/Automated_Vehicles_ Policy.pdf. Last Accessed 12 Sept 2014

2. Heart of Atlanta Motel Inc. v. United States (1964), 379 U.S. 241

3. Swanson A (2014) "Somebody grab the wheel!" state autonomous vehicle legislation and the road to a national regime. Marq L Rev 97: 1085

4. US v. Knotts, 460 U.S. 276 (1983)

5. US v. Jones, 565 U.S. (2012)

6. Riley v. California, 573 U.S. _ (2014)

7. Anderson JM, Kalra N, Stanley KD, Sorensen P, Samaras C, Oluwatola O (2014) Autonomous vehicle technology: a guide for policymakers. RAND Corporation, Santa Monica, CA, RR-443-1-RC, pp. 12-36 